\title{
PROBING THE SURFACE-VACUUM INTERFACE WITH SPIN-SENSITIVE METASTABLE ATOM DEEXCITATION, ELECTRON CAPTURE AND ELECTRON EMISSION SPECTROSCOPIES
}

\author{
G. K. WALTERS AND C. RAU \\ Physics Department and Rice Quantum Institute, Rice University, Houston, TX 77251-1892
}

\begin{abstract}
Spin-Polarized Metastable Atom Deexcitation (SPMDS) and Electron Capture (ECS) Spectroscopies probe the exponential tails of electronic wavefunctions extending from the surface into the vacuum, and are consequently extremely sensitive to the surface-vacuum interface. The use of SPMDS to probe the near-surface vacuum magnetization of $\mathrm{Ni}(110)$ and $\mathrm{Fe}(110)$ and the dramatic changes that result upon exposure to ambient gases is discussed, as is the use of ECS and Spin-Polarized Electron Emission Spectroscopy (SPEES) to determine the ferromagnetic and critical behavior of surfaces and ultra-thin epitaxial systems.
\end{abstract}

\section{INTRODUCTION}

Particle (atoms, ions)-surface scattering experiments provide a powerful means to study the topmost surface-layer electronic and magnetic properties of magnetic materials. This can be achieved by keeping the energy component $E_{\perp}$ of the incident particles normal to the probed surface below $10-20 \mathrm{eV}$, thus preventing their penetration into the surface and assuring top layer specificity in their interaction with target surfaces. Spin-polarized metastable (atom) deexcitation spectroscopy (SPMDS), electron capture spectroscopy (ECS), and spin-polarized electron emission spectroscopy (SPEES) have emerged as extremely surface specific probes of magnetic properties at the surface-vacuum interface. 1 The physical processes underlying each of these spectroscopies are briefly described below and selected experimental results are presented to illustrate the insights into surface magnetic behavior that they provide.

\section{SPIN-POLARIZED METASTABLE DEEXCITATION SPECTROSCOPY (SPMDS)}

In SPMDS surface electronic and magnetic structure and the near-surface magnetic environment are probed by investigating spin dependences in the interaction of thermal-energy $(\sim 0.03 \mathrm{eV})$ electron-spin-polarized $\mathrm{He}\left(2^{3} \mathrm{~S}\right)$ metastable atoms with a magnetized surface. The energy distributions and polarization of electrons ejected from the surface as a result of metastable-atom deexcitation are measured as is (for a magnetized surface) any spin dependence in the total ejected-electron signal. ${ }^{2,3}$

The apparatus is shown schematically in Fig. 1 and is described in detail elsewhere. ${ }^{2-4}$ Briefly, a fraction of the atoms contained in a ground-state helium atom beam are collisionally excited to the $2^{1,3} \mathrm{~S}$ levels by a coaxial electron beam. The $2^{1} \mathrm{~S}$ atoms are removed from the beam by illuminating it with $2.06-\mu \mathrm{m}$ radiation from a helium discharge which excites $2^{1} \mathrm{~S} \rightarrow$ $2^{1} \mathrm{P} \rightarrow 1^{1} \mathrm{~S}$ transitions. A weak $(\sim 0.5 \mathrm{G})$ magnetic field is applied perpendicular to the beam to preserve a well-defined quantization axis. Circularly polarized $1.08-\mu \mathrm{m} 2^{3} \mathrm{~S} \rightarrow 2^{3} \mathrm{P}$ resonance radiation from a high-power rf-excited helium lamp is incident along the magnetic field direction and is used to optically pump the ${ }^{3} \mathrm{~S}$ atoms to increase the relative populations in the $\mathrm{M}_{\mathbf{J}}\left(\mathrm{M}_{\mathbf{S}}\right)=+1$ or -1 magnetic sublevels. The resultant beam polarization is defined as

$$
P_{H e}=\frac{F_{+}-F_{-}}{F_{+}+F_{0}+F_{-}}
$$




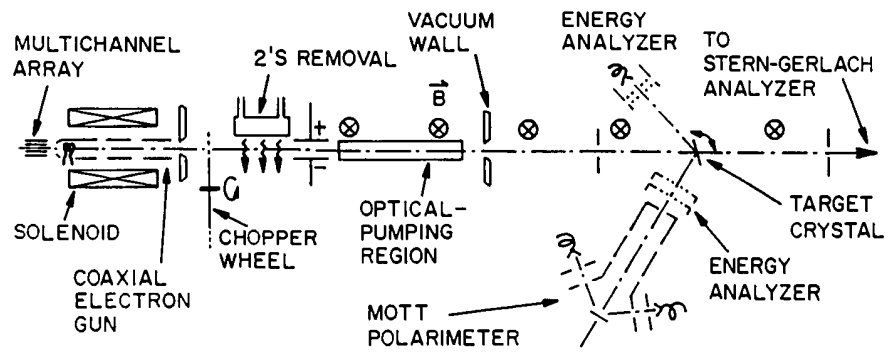

Fig. 1. Schematic diagram of the SPMDS apparatus.

where $F_{+}, F_{0}$, and $F_{-}$are the fluxes of $\mathrm{He}\left(2^{3} S\right)$ atoms with $M_{S}=+1,0$ and -1 , respectively. The beam polarization, $\mathrm{P}_{\mathrm{He}} \sim 0.4$, is measured by a Stern-Gerlach analyzer ${ }^{-4}$ and can be simply reversed $\left(\mathrm{P}_{\mathrm{He}} \rightarrow-\mathrm{P}_{\mathrm{He}}\right)$ by changing the sense of circular polarization of the optical pumping radiation.

The energy distribution of the electrons ejected from the target surface is measured using a simple retarding grid energy analyzer, and those electrons with sufficient energy to overcome the retarding potential barrier are detected by a channeltron. The number of electrons with energies in some particular interval is determined by switching the potential applied to the retarding grid between the appropriate limits and observing the resultant change in the detected electron signal. The energy analyzer is also used to investigate spin dependences in the total number of electrons ejected with energies greater than the cut off determined by the potential $\mathrm{V}$ applied to the retarding grid. Such spin dependences are characterized by an asymmetry parameter $A(V)$ defined as

$$
A(V)=\frac{1}{\left|P_{\mathrm{He}}\right|} \frac{\mathrm{I}_{+}-\mathrm{I}_{-}}{\mathrm{I}_{+}+\mathrm{I}_{-}}
$$

where $\mathrm{I}_{+}$and $\mathrm{L}_{-}$are the ejected electron currents observed with the incident beam polarized parallel and antiparallel, respectively, to the majority spin direction in the (magnetized) target.

A second independent experimental parameter, the polarization of the ejected electrons, can also be measured. This is accomplished using a compact Mott polarimeter equipped with a retarding potential energy analyzer. 5 The average polarization of those ejected electrons with energies greater than the cut off set by the retarding potential $V$ applied in the energy analyzer is determined by measuring the asymmetry in the count rates of electrons quasielastically scattered (at $20 \mathrm{keV}$ ) through $\pm 120^{\circ}$ at a gold target. The electron polarization, which depends both on the helium atom polarization and the retarding potential, is defined by

$$
P_{e}\left(P_{H e}, V\right)=\frac{I_{P}-I_{A}}{I_{P}+I_{A}}
$$

where $I_{P}$ and $I_{A}$ are the currents of electrons ejected with spins parallel and antiparallel, respectively, to the majority spin direction in the target.

Values of the asymmetry A(V) measured for an atomically clean $\mathrm{Ni}(110)$ surface $^{2}$ and for an $\mathrm{Fe}(110)$ surface with residual carbon $(\sim 9 \%)$ and oxygen $(\sim 4 \%)^{3}$-- the cleanest achievable and henceforth referred to as the "clean" Fe surface .. are shown in Figs. 2 and 3. Fig. 3 also 


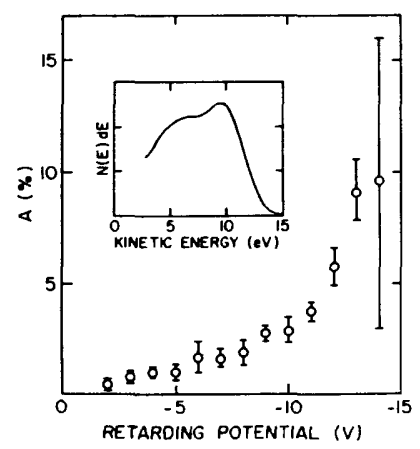

Fig. 2. Polarization asymmetry $\mathrm{A}$ as a function of retarding potential for a clean, magnetized $\mathrm{Ni}(110)$ sample at $\sim 130^{\circ} \mathrm{C}$. The inset shows the measured secondary-electron energy distribution.

shows the changes in $A(V)$ that result when the $\mathrm{Fe}(110)$ surface is exposed to oxygen. The ejected electron energy distributions for the clean surfaces, and for $\mathrm{Fe}(110)$ following $8 \mathrm{~L}$ exposure to oxygen, are shown in insets. Fig. 3 also includes values of the asymmetry expected for an atomically clean $\mathrm{Fe}(110)$ surface obtained by linear extrapolation of the asymmetries measured at different oxygen coverages as inferred from Auger analysis. (Tests revealed that the presence of small amounts of carbon on the surface had a negligible effect on the measured asymmetries.)

The asymmetries shown in Figs. 2 and 3 can be interpreted in terms of the theory of spin polarized metastable atom deexcitation at magnetic surfaces recently developed by Penn and Apell (PA). ${ }^{6} \mathrm{~A} \mathrm{He}\left(2^{3} S\right.$ ) atom incident upon a clean, relatively high-workfunction surface such as $\mathrm{Ni}(110)$ or $\mathrm{Fe}(110)$ first undergoes resonance ionization (RI) in which the excited $2 \mathrm{~s}$ electron tunnels into an unfilled level above the Fermi surface. The resulting $\mathrm{He}^{+}$ion continues toward the surface where it undergoes Auger neutralization (AN) in which a conduction electron from the metal tunnels into the $1 \mathrm{~s}$ hole, the released energy being communicated to a second (Auger) conduction electron which may escape from the metal. The energy available to the escaping electron depends on the energy of the $\mathrm{He}^{+} 1 \mathrm{~s}$ hole and this decreases as the ion approaches the surface due to the $\mathrm{He}^{+}$image potential. Thus the farther from the surface that the AN event occurs, the greater the energy (on the average) of the escaping electron. PA show that the
Fig. 3. Values of the asymmetry $A(V)$ for (a) an atomically clean $\mathrm{Fe}(100)$ surface (obtained by extrapolation, see text); (b) a sputtered and annealed $\mathrm{Fe}(110)$ surface; and (c), (d), (e), (f), and (g) an $\mathrm{Fe}(110)$ surface following exposure to 1, 2, 4,8 , and $16 \mathrm{~L}$ of oxygen, respectively. The inset shows the ejected electron-energy distributions for a clean $\mathrm{Fe}(110)$ surface ( ---$)$ and following an 8-L exposure to oxygen ( $\longrightarrow$ ). 
measured asymmetries arise as a consequence of a non-zero magnetization in the vacuum well outside the surface where AN occurs.

The AN rate at some distance $\mathrm{z}$ from the target surface is proportional to the number density of conduction electrons at $\mathrm{z}$ that are available to fill the $\mathrm{He}^{+} 1 \mathrm{~s}$ hole. For incident $\mathrm{He}\left(2^{3} \mathrm{~S}\right)$ atoms, and hence $\mathrm{He}^{+}$ions, with positive (negative) polarizations (i.e., spins parallel (antiparallel) to the majority spin direction in the magnetized target) neutralization can only occur with minority (majority) conduction electrons (the helium ground state is a spin singlet). Thus the average distance at which AN occurs, and hence the average energy available to the Auger electron, will depend on the spin orientation of the incident $\mathrm{He}\left(2^{3} \mathrm{~S}\right)$ atoms because the majority and minority spin densities in the vacuum are in general unequal for a ferromagnetic target.

Using this model, PA have shown that the sign of the asymmetry parameter $A(V)$ will be positive (negative) if the conduction electron density in the vacuum outside the target surface is predominantly minority (majority). Their analysis of the $\mathrm{Ni}(110)$ asymmetry yields a magnetization of $\sim-20 \%$ at the Fermi energy and $4.5 \AA$ from the surface. ${ }^{6}$ The negative magnetization in the vacuum is consistent with band calculations of Wimmer et al. who show that the nickel $s-p$ electrons, which through $s-d$ hybridization are polarized oppositely from the $d$ electrons that dominate the total magnetic moment in the bulk, spill out into the vacuum region and are dominant beyond $\sim 2.5 \AA .7$ Negative magnetization in the vacuum above $\mathrm{Ni}(110)$ has also been reported by Rau, based on electron capture experiments. ${ }^{8}$ Exposure of the Ni(110) surface to 4 Langmuirs of $\mathrm{CO}$ reduces the measured asymmetry to zero, suggesting that the vacuum magnetization is quenched to $\leq \pm 2 \%$ within experimental uncertainty. ${ }^{2}$

The data in Figure 3 show that $A(V)>0$ for a clean $F e(110)$ surface, requiring on the basis of the PA theory that, as for $\mathrm{Ni}(110)$, minority electrons are dominant at distances $\sim 3-5 \AA$ outside the surface where AN occurs. However, the measured asymmetry is extremely sensitive to the presence of oxygen on the surface, reversing sign and becoming strongly negative for exposures above about $4 \mathrm{~L}$. According to the PA theory this requires that the vacuum magnetization at distances where AN occurs change from negative to positive upon oxygen adsorption. This result is consistent with subsequent $a b$ initio electronic structure calculations by $\mathrm{Wu}$ and Freeman, which reveal that the $\mathrm{Fe}(110)$ vacuum magnetization at distances where AN occurs is indeed negative, strongly so for states near the Fermi energy that are responsible for the large asymmetry observed at the highest ejected electron energies. ${ }^{9}$ The calculations also agree with experiment in showing that the vacuum magnetization is positive for the $\mathrm{O} / \mathrm{Fe}(110)$ surface.

The polarizations $\mathrm{P}_{\mathrm{e}}\left(\mathrm{P}_{\mathrm{He}}, \mathrm{V}\right)$ of electrons ejected from clean and oxygen-exposed $\mathrm{Fe}(110)$ surfaces were also measured. The data are summarized in Table I for several values of the retarding potential $\mathrm{V}$. Table I includes data obtained using an unpolarized incident beam, and an incident beam polarized either parallel or antiparallel to the majority spin direction in the (magnetized) sample. Since the polarizations of both the incident $\mathrm{He}\left(2^{3} S\right)$ atoms and ejected electrons are defined relative to the target majority spin direction, the measured values of $P_{e}$ should remain unchanged upon reversal of the magnetization of the target $(M \uparrow \rightarrow M \downarrow)$, as is observed. In contrast to the marked sensitivity of the asymmetry $A(V)$ to oxygen exposure, both the ejected-electron energy distribution and polarization of electrons ejected by an unpolarized incident $\mathrm{He}\left(2^{3} \mathrm{~S}\right)$ beam are essentially unchanged by oxygen exposure.

The data in Table I also reveal a significant spin correlation favoring the ejection of electrons with the same spin orientation as the incident metastable atoms. This spin correlation, which amounts to $\sim 20 \%$, i.e., the change in polarization of the ejected electrons is $\sim 20 \%$ that of the incident atoms, is similar to that observed from paramagnetic targets ${ }^{10}$ for which it has been demonstrated that metastable deexcitation occurs via RI+AN exclusively. ${ }^{11}$ Indeed, this unexpected $\gtrsim 20 \%$ spin correlation in Auger neutralization of $\mathrm{He}^{+}$ions at surfaces has been observed for every target investigated, both paramagnetic and ferromagnetic.

The ejected electron polarizations resulting from the deexcitation of an unpolarized $\mathrm{He}\left(2^{3} \mathrm{~S}\right)$ beam at both a clean $\mathrm{Fe}(110)$ surface, and following oxygen exposure, are positive, i.e., the ejected electron polarization is parallel to the majority spin direction and reflects the conduction band polarization. This requires that, on average, the ejected electrons originate 
TABLE I. Measured spin polarization $P_{e}(V)$ of electrons ejected by both unpolarized $\mathrm{He}\left(2^{3} S\right)$ atoms and by $\mathrm{He}\left(2^{3} S\right)$ atoms polarized parallel and antiparallel to the majority-spin direction in the target. Data for the clean $\mathrm{Fe}(110)$ surface after reversing the target magnetization $(M \uparrow \rightarrow M \downarrow)$ are included. The majority-spin direction in the target is taken to be the direction of positive $P_{\mathrm{He}}$ and $P_{\mathrm{e}}(V)$. The uncertainty in each measured value of $P_{e}$ is $\sim \pm 0.03$.

\begin{tabular}{|c|c|c|c|c|c|c|c|}
\hline & $\boldsymbol{V}$ & -0.40 & $\begin{array}{l}M \uparrow \\
P_{\mathrm{He}} \\
0 \\
P_{e}(V)\end{array}$ & +0.40 & -0.40 & $\begin{array}{c}M \downarrow \\
P_{\mathrm{He}} \\
0 \\
P_{e}(V)\end{array}$ & +0.40 \\
\hline (a) Clean $\mathrm{Fe}(110)$ & $\begin{array}{l}0 \\
3 \\
6 \\
9\end{array}$ & $\begin{array}{r}0.07 \\
0.02 \\
-0.01 \\
-0.06\end{array}$ & $\begin{array}{l}0.12 \\
0.08 \\
0.07 \\
0.10\end{array}$ & $\begin{array}{l}0.21 \\
0.18 \\
0.14 \\
0.22\end{array}$ & $\begin{array}{l}0.004 \\
0.04 \\
0.02 \\
0.02\end{array}$ & $\begin{array}{l}0.10 \\
0.09 \\
0.04 \\
0.11\end{array}$ & $\begin{array}{l}0.21 \\
0.15 \\
0.19 \\
0.21\end{array}$ \\
\hline (b) $\mathrm{O} / \mathrm{Fe}(110)$ (8-L exposure) & $\begin{array}{l}0 \\
3 \\
6 \\
9 \\
\end{array}$ & $\begin{array}{r}0.04 \\
-0.03 \\
-0.02 \\
-0.03\end{array}$ & $\begin{array}{l}0.10 \\
0.07 \\
0.08 \\
0.09\end{array}$ & $\begin{array}{l}0.15 \\
0.14 \\
0.15 \\
0.21\end{array}$ & & & \\
\hline
\end{tabular}

deeply enough within the target surface that their polarizations are not significantly affected by the presence of an oxygen adlayer. Photoemission 12 and electron bombardment ${ }^{13}$ studies of magnetized iron surfaces also have shown that the ejected electron polarization mirrors the band polarization, but the measured polarizations are much greater than observed in the present work. The present data are, however, in reasonable agreement with the results of Kirschner et al. who studied the polarization of electrons ejected (by potential ejection) when $1 \mathrm{keV} \mathrm{He}^{+}$or $\mathrm{Ar}^{+}$ions are neutralized at a magnetized $\mathrm{Fe}(110)$ surface. ${ }^{14}$ They attribute the generally low polarizations of the ejected electrons to a reduction in the band polarization in the near surface region, where the ejected electrons originate, because of the spill-out of negatively polarized s-p electrons above the surface. Alternately, matrix element effects favoring Auger ejection from the s-p band could account for the low measured polarizations.

In summary, measurements and analysis of asymmetry and spin-polarization of electrons ejected from magnetic surfaces by $\mathrm{He}\left(2^{3} \mathrm{~S}\right)$ metastable-atom deexcitation demonstrate that SPMDS is an extraordinarily sensitive probe of the surface magnetic environment and the pronounced changes that can result upon exposure to ambient gases.

\section{ELECTRON CAPTURE AND SPIN-POLARIZED ELECTRON EMISSION SPECTRO- SCOPIES}

Electron capture spectroscopy (ECS) and spin-polarized electron emission spectroscopy (SPEES) utilize capture and emission of spin-polarized electrons during grazing-angle surface reflection of fast ions at magnetic surfaces. These spectroscopies provide a powerful means for study of two-dimensional (2D) magnetic properties (critical behavior, element-specific ferromagnetic order, magnetic anisotropies, etc.) both of bulk surfaces and of ultra-thin films, with unprecedented surface sensitivity. 15,16

The fundamental physical process in ECS is the capture of one or two spin-polarized electrons during small-angle surface reflection of fast ions, enabling the study of both longrange and short-range order at surfaces of ferromagnetic materials. ${ }^{17,18}$ For $150 \mathrm{keV}$ deuterons and for an ion angle of incidence of $0.2^{0}$ the distance of closest approach of the ions to the reflecting surface is about $0.1 \mathrm{~nm}$ (see Fig. 4, full line). Therefore, the ions probe spin-polarized local electron densities of state at the topmost surface layer. For the measurement of long-range surface ferromagnetic order, one electron capture processes $\left(D^{+}+e^{-}=D^{0}\right)$ are exploited by determining the spin polarization of the captured electrons; details are presented elsewhere. For the measurement of short-range ferromagnetic order, protons or deuterons are used to study 
two-electron capture processes (e.g., $\mathrm{D}^{+}+2 \mathrm{e}^{-}=$ D). ${ }^{17}$ The only stable bound state of $\mathrm{H}^{-}$or $\mathrm{D}^{-}$ is the $1 \mathrm{~s}^{2}{ }^{1} \mathrm{~S}$ state. Therefore, stable $\mathrm{H}^{-}$or $\mathrm{D}^{-}$ ions can only be formed by capture of two electrons with oppositely oriented spins. In the ESP experiments reported here, $25 \mathrm{keV}$ protons or $150 \mathrm{keV}$ deuterons are used. For this energy range, the distance within which 2 electrons are captured by a single ion is in the range 0.5-2 $\mathrm{nm}$. Thus, two-electron capture processes are sensitive to short-range ferromagnetic order existing within a range of only a few atomic neighbors. It is obvious that two-electron capture will be strongly suppressed by the presence of short-range ferromagnetic order where predominantly electrons with parallel oriented spins are available for capture by a single ion. The reduction in the $\mathrm{H}^{-} / \mathrm{H}^{+}$or $\mathrm{D}^{-}$ $/ \mathrm{D}^{+}$ratio measured after beam reflection at the

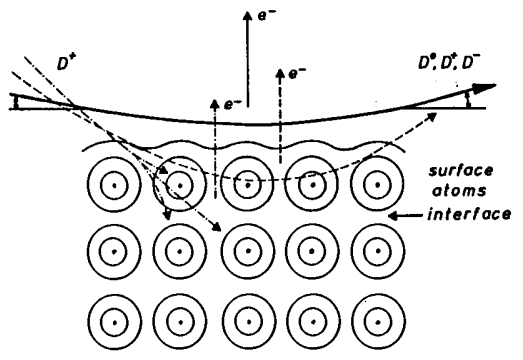

Fig. 4. Scheme illustrating ion trajectories for various scattering angles, the emission of ioninduced electrons and the surface potential plotted on a plane perpendicular to the reflecting surface. surface of a magnetic sample, relative to that for a nonmagnetic target such as $\mathrm{Cu}$, provides a direct measure of the short-range ESP at a magnetic surface.

In angle- and energy-resolved, spin-polarized electron emission spectroscopy (SPEES), small angle surface scattering of energetic $(5-150 \mathrm{keV})$ ions $\left(\mathrm{H}^{+}, \mathrm{He}^{+}\right.$or $\left.\mathrm{Ne}^{+}\right)$is used to study the emission of spin-polarized electrons as a measure of long-range surface ferromagnetic order. Fig. 1 illustrates ion trajectories for various scattering angles, the ion-induced emission of electrons, and the surface potential plotted on a plane perpendicular to the reflecting surface. Varying the ion scattering angle from $0.2^{\circ}$ up to $45^{\circ}$ results in an increase of the probing depth of the incident ions from the topmost surface layer to interface and deeper layers. Electrons emitted along the surface normal (emission cone angle $8^{\circ}$ ) of a nonmagnetic or remanently magnetized target are both energy- and spin analyzed.

Ultra-thin magnetic films are deposited by electron beam evaporation. The thickness of the films is determined with a calibrated quartz oscillator, calibrated Auger electron signals and with RHEED oscillations. The island-free growth of the films is checked by monitoring the ion reflectivity and the energy distribution of the specularly reflected deuterons. At the surface of all films studied so far, the reflectivity is not reduced from its initial value of $95 \%$ measured at the carefully prepared substrate surfaces which have been shown by scanning tunneling microscopy to be atomically flat over distances of $150-200 \mathrm{~nm}$. Furthermore, the presence of islands would yield an additional energy loss of the deuterons caused by penetration of the ions through islands by planar channeling, which is not observed.

Ultra-thin $(5 \mathrm{~nm})$ hcp $\mathrm{Tb}(0001)$ films which are epitaxially and homogeneously deposited at $300 \mathrm{~K}$ on bcc W(110) substrates provide a striking example of the use of ECS for studies of long-range ferromagnetic order with top layer specificity. 19 The electron spin polarization (ESP) of the captured electrons is measured. Their polarization

$$
P=\frac{n_{+}-n_{-}}{n_{+}+n_{-}}
$$

is defined relative to the direction of the magnetic field $\mathrm{H}$ applied at the target, and $\mathrm{n}^{+}$and $\mathrm{n}^{-}$are the numbers of majority-and minority-spin electrons detected. ${ }^{17}$ In the case of rare-earth metals such as $\mathrm{Tb}$ and $\mathrm{Gd}$, the measured polarization $\mathrm{P}$ is attributed to the polarization of the $5 \mathrm{~d}-6 \mathrm{~s}$ surface electrons ${ }^{17}$ which is proportional to the surface magnetization of the localized $4 \mathrm{f}$ 


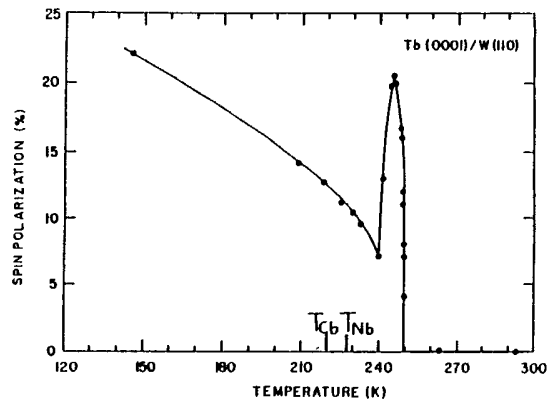

Fig. 5 Electron spin polarization $\mathrm{P}(\%)$ of the topmost surface layer of $5 \mathrm{~nm}$ thin $\mathrm{Tb}(0001) / \mathrm{W}(110)$ films as function of temperature. $T_{\mathrm{Cb}}$ denotes the bulk Curie temperature, and $\mathrm{T}_{\mathrm{Nb}}$ denotes the bulk Neel temperature of $\mathrm{Tb}$.

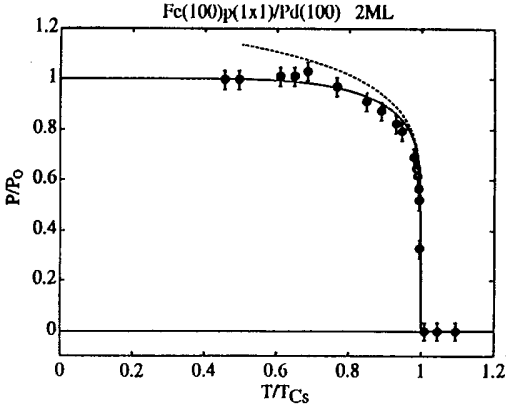

Fig. 6. Electron spin polarization $\mathrm{P} / \mathrm{P}_{\mathrm{o}}$ as function of $\mathrm{T} / \mathrm{T}_{\mathrm{Cs}}$ for the surface of a $2 \mathrm{ML}$ thin bct $\mathrm{Fe}(100) \mathrm{p}(1 \times 1) / \mathrm{Pd}(100)$ film. The solid and dashed lines represent, respectively, the exact solution of the 2D Ising model and the power law approximation for $\mathrm{T} \rightarrow \mathrm{T}_{\mathrm{Cs}}$.

electrons, the predominant carriers of the magnetization. $\mathrm{P}$ is measured in magnetic fields ranging between 25 and $600 \mathrm{Oe}$, the samples being magnetized along the W[100] direction and the temperature being kept constant within $0.02^{\circ}$. Such applied fields have been shown to have a negligible effect on the electron spin polarization in the investigated temperature range. ${ }^{20}$

In Fig. 5, the temperature dependence of $\mathrm{P}$ at $\mathrm{Tb}(0001) / \mathrm{W}(110)$ surfaces is shown for $\mathrm{H}=$ $250 \mathrm{Oe}$. H was varied between $25 \mathrm{Oe}$ and $600 \mathrm{Oe}$, and no significant influence on the polarization data was detected. Nonzero $P$ values establish that the surfaces of the films are ferromagnetically ordered up to $248 \mathrm{~K}$, which lies above both the bulk Curie and Néel temperatures, $\mathrm{T}_{\mathrm{Cb}}$ and $\mathrm{T}_{\mathrm{Nb}}$ as indicated in Fig. 5. With increasing temperature, $\mathrm{P}$ decreases from $22 \%$ at $146 \mathrm{~K}$, reaching a value of $7 \%$ at about $240 \mathrm{~K}$, which lies slightly above $\mathrm{T}_{\mathrm{Cb}}$ and $\mathrm{T}_{\mathrm{Nb}}$. As T increases further, $\mathrm{P}$ increases very steeply to $21 \%$ at $243 \mathrm{~K}$, then drops suddenly to zero at a surface Curie temperature $\mathrm{T}_{\mathrm{Cs}}=249.96 \mathrm{~K}$. In more recent $\mathrm{ECS}$ experiments, it has been found that the short-range ESP extends up to temperatures of about $300 \mathrm{~K}$.

From the measured temperature variation of $P$ the critical exponent $B$ is determined simultaneously with $T_{C s}$ by a linear least-square fit. For $\left(T_{C s}-T\right) / T_{C s}$ ranging between $2 \times 10^{-2}$ and $10^{-4}$, a value $B=0.348 \pm 0.01$ is obtained.

Previous magnetic studies of surface critical behavior have been restricted to the case $T_{C s}=$ $T_{\mathrm{Cb}}$, for which $\mathrm{B}$ has been measured to be $0.75 \pm 0.05^{21}$ for several systems. The only other experimental determination of $B$ for the case $T_{C s}$ larger than $T_{C b}$ was for ultra-thin films of $\mathrm{V}(100)$ on $\mathrm{Ag}(100)$ substrates, ${ }^{22}$ for which $\mathrm{T}_{\mathrm{Cb}}=0$. For that system ECS measurements yielded $\beta=0.125$, a value identical to that of the two-dimensional Ising ferromagnet. In the present case, $\mathrm{T}_{\mathrm{Cs}}$ lies close to $\mathrm{T}_{\mathrm{Cb}}$, and one does not expect 2D Ising critical behavior. The fact that $\mathrm{T}_{\mathrm{Cs}} \neq \mathrm{T}_{\mathrm{Cb}}$ indicates that the magnetic couplings between the surface spins are strongly anisotropic, which may explain why the measured $\beta=0.348$ is much smaller than the value $\beta=$ 0.75 expected in the absence of anisotropy. ${ }^{23}$

In other ECS experiments, ultra-thin (1-4 ML) bct $\mathrm{Fe}(100)$ films were deposited on atomically flat $\mathrm{Pd}(100)$ substrates. For a substrate temperature of $293 \mathrm{~K}$ and an evaporation rate of $0.002 \mathrm{~nm} / \mathrm{s}$, homogeneous and island-free growth of the Fe films is obtained. ${ }^{24}$ The samples are magnetized in fields up to $75 \mathrm{Oe}$, the temperature of the samples being kept constant within $0.05^{\circ}$. Such applied fields have a negligible effect on the ESP in the investigated temperature range and it was found that, within experimental errors, the remanent magnetization is equal to the saturation magnetization. Fig. 6 shows the normalized long-range electron spin polarization 
$\mathrm{P} / \mathrm{P}_{\mathrm{O}}$ at the surface of a $2 \mathrm{ML} \mathrm{Fe}(100)$ film on $\operatorname{Pd}(100)$, as a function of $\mathrm{T} / \mathrm{T}_{\mathrm{Cs}}$, with $\mathrm{T}_{\mathrm{Cs}}=$ $613.1 \mathrm{~K}$ being the measured surface Curie temperature and $P_{0}=-33 \%$ the calculated electron spin polarization at $T=0$. The full line in Fig. 6 represents the temperature dependence of the magnetization as predicted by Yang for the $2 \mathrm{D}$ Ising model, 25 and the dashed line gives the best fit in the asymptotic power law approximation with $\beta$ $=0.125$. It is obvious that the experimental data are precisely described by the exact solution of the 2D Ising model given by Yang.

For the SPEES experiments discussed here, surface scattering of $25 \mathrm{keV} \mathrm{Ne}{ }^{+}$ions was used to study the emission of spin-polarized electrons produced as a consequence of the particle-surface interaction. Figure $7 \mathrm{a}$ shows for an angle of incidence $\alpha=1^{\circ}$ the energy distribution (relative to the vacuum level) and the ESP of electrons emitted along the surface normal from clean (solid line) and $\mathrm{O}$-covered (dashed line) polycrystalline $\mathrm{Fe}$ surfaces. ${ }^{19}$ At this incidence angle the $\mathrm{Ne}^{+}$ions are specularly reflected and do not penetrate the $\mathrm{Fe}$ surfaces. For both clean $\mathrm{Fe}$ and $\mathrm{O} / \mathrm{Fe}$, the energy distribution of the emitted electrons peaks at around $4 \mathrm{eV}$. However at $\mathrm{O} / \mathrm{Fe}$ a strong increase in the intensity of emitted electrons is found as compared to that of clean Fe.

The ESP of electrons emitted from the topmost surface layer of clean $\mathrm{Fe}$ is $\mathrm{P}=(33 \pm 2) \%$ for $\mathrm{E}=10 \mathrm{eV}$ but increases to $\mathrm{P}=(48 \pm 2) \%$ for $\mathrm{E}=4 \mathrm{eV}$. These values are far above the $28 \%$ average bulk magnetization value of $\mathrm{Fe}$. For $\mathrm{Fe}$ surfaces with one monolayer of $\mathrm{O}$, the average ESP of electrons at $\mathrm{E}=10 \mathrm{eV}$ remains nearly unchanged $(32 \pm 2) \%$, but for electrons with $\mathrm{E}=4 \mathrm{eV}$ the ESP drops from $(48 \pm 2) \%$ to $-(14 \pm 2) \% \mathrm{eV}$ indicating the presence of a magnetically active surface layer.

It is of considerable interest to determine whether the measured ESP of the emitted electrons reflects the layer-dependent net magnetization of a material. From ion-16,25,26 and electron-induced electron spectra, 27 there is evidence that the ESP of electrons emitted at high energies $(\approx 10 \mathrm{eV}$ above the vacuum level) scales roughly with the average net magnetization.

For $\alpha=8^{\circ}, \mathrm{E}_{\perp}$ is $32.8 \mathrm{eV}$, and the ions can penetrate the topmost surface layer and excite electrons from the second layer. The shape of the energy distributions of the emitted electrons are similar to those of Fig. 7a with the peak maximum shifted upwards to about $5 \mathrm{eV}$ (see Fig. 7b). In this case the average ESP of the electrons emitted from clean $\mathrm{Fe}$ increases from $\mathrm{P}=29 \%$ at $\mathrm{E}=10 \mathrm{eV}$ to $\mathrm{P}=50 \%$ at $\mathrm{E}=4 \mathrm{eV}$. For the
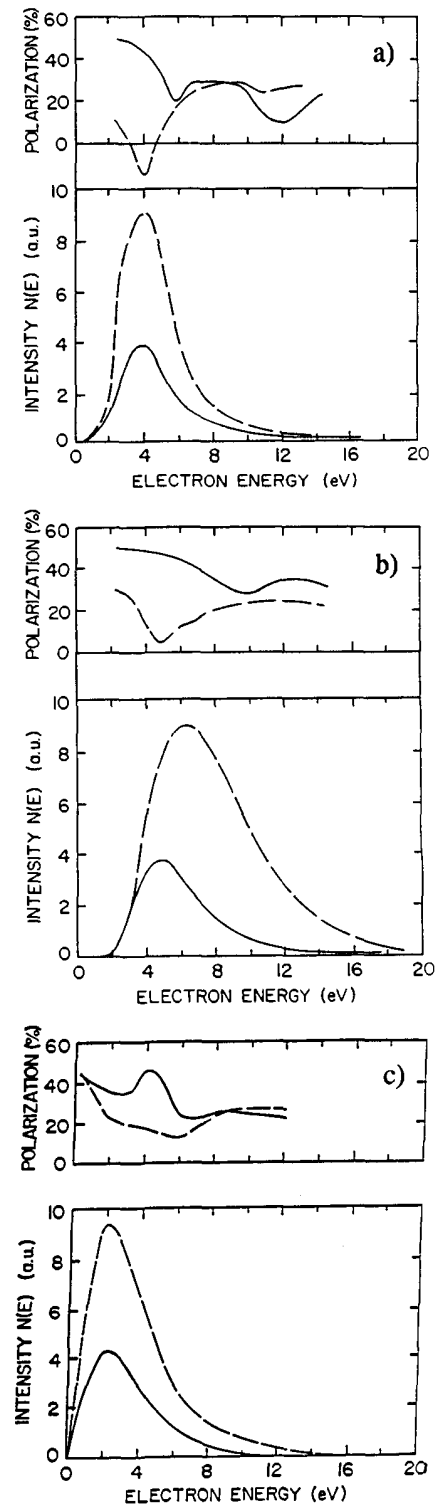

Fig. 7. Energy distribution and ESP as function of the energy $E$ of electrons emitted from clean (solid line) and O-covered (dashed line) $\mathrm{Fe}$ surfaces by incident $25 \mathrm{keV} \mathrm{Ne}{ }^{+}$ions and with incidence angles of $1^{\circ}$ (a), $8^{\circ}$ (b) $45^{\circ}$ (c). 
$\mathrm{O} / \mathrm{Fe}$ surface the average ESP of electrons with $\mathrm{E}=10 \mathrm{eV}$ remains unchanged $(32 \pm 2 \%)$, whereas for electrons with $\mathrm{E}=4 \mathrm{eV}$ the ESP decreases from $(50 \pm 2) \%$ to $(14 \pm 2) \% \mathrm{eV}$.

Fig. 7c shows for the energy distributions and the ESP's of electrons emitted from clean (solid line) and $\mathrm{O} / \mathrm{Fe}$ (dashed line) surfaces for $\alpha=45^{\circ}$. For this incidence angle $\mathrm{E}_{\perp}$ is $12.5 \mathrm{keV}$ and the ions can penetrate deeply into the solid and excite electrons from bulk layers. In this case the energy distributions of clean and O-covered Fe surfaces are similar to those obtained in electron-induced secondary electron emission experiments, ${ }^{26-28}$ peaking at $2 \mathrm{eV}$. For $\mathrm{O} / \mathrm{Fe}$ surfaces, however, a strong increase in the intensity of the emitted electrons is again observed. For electrons excited in bulk layers electron cascading and multiple scattering are the dominant processes occurring during electron transport to the surface, which causes the well-known $2 \mathrm{eV}$ peak in electron- or ion-induced electron spectra. This is consistent with the data for $\alpha=1^{\circ}$ and $\alpha=8^{\circ}$ where the energy distributions of the emitted electrons peak at higher energies (around 4-5 $\mathrm{eV}$ ) showing that electron cascading and multiple scattering processes are less pronounced. As regards the average ESP of the electrons emitted from clean $\mathrm{Fe}$, an increase from $\mathrm{P}=(25 \pm 2) \%$ for $\mathrm{E}=10 \mathrm{eV}$ to $\mathrm{P}=(45 \pm 2) \%$ for $\mathrm{E}=4 \mathrm{eV}$ is observed. For the $\mathrm{O} / \mathrm{Fe}$ surface, the average ESP of electrons with $\mathrm{E}=10 \mathrm{eV}$ remains unchanged $(25 \pm 2 \%)$, whereas for electrons with $\mathrm{E}=4 \mathrm{eV}$ the ESP drops to (15 \pm 2$) \% \mathrm{eV}$.

Changing $\alpha$ from $45^{\circ}$ to $1^{\circ}$, which corresponds to a reduction in the probing depth from deep lying layers, where bulk physical properties are probed, to the topmost surface layer, results in an increase in the ESP of "high-energy" electrons from $25 \%$ to $33 \%$. This would imply that for polycrystalline $\mathrm{Fe}$ surfaces the net magnetization increases in going from the bulk to the surface. Assuming that the ESP of electrons emitted at high energies $(\approx 10 \mathrm{eV})$ scales roughly with the average net magnetization, it is tempting to interpret this surface enhancement of the ESP in terms of theoretically predicted magnetic surface states which cause enhancements of the magnetization at $\mathrm{Fe}(100)$ and $\mathrm{Fe}(110)$ surfaces. ${ }^{29,30}$

For low emitted electron energies, the measured ESP's from clean Fe are substantially enhanced above the bulk polarization values, as has been observed also for electron- and ioninduced emission. This enhancement can be attributed to Stoner excitations across the ferromagnetic exchange gap which occur during inelastic exchange scattering of minority electrons. ${ }^{31,32}$ The fact that the enhancements are observed to be approximately the same for electrons emitted from the surface layer $\left(\alpha=1^{\circ}\right)$ and from subsurface and deeper layers $\left(\alpha=8^{\circ}\right.$ and $45^{\circ}$ ) suggests that the mean free path for Stoner excitation is of order one monolayer or less.

Finally, the substantial polarizations of electrons emitted from $\mathrm{O} / \mathrm{Fe}$, and the pronounced dependence of the ESP at low energies on the incidence angle of the ion beam clearly demonstrate the absence of a magnetically dead layer at the surface and are consistent with the existence of spin-split electronic bands in the occupied and unoccupied parts of the band structure of $\mathrm{O} / \mathrm{Fe}$ surfaces. 33

In conclusion, the experiments discussed here provide clear evidence that ECS and SPEES are powerful techniques for the study of topmost and interface layer magnetic properties.

This work was supported by the National Science Foundation, the Department of Energy, the Welch Foundation and the Texas Higher Education Coordinating Board.

\section{References}

1. F.B. Dunning, C. Rau and G.K. Walters, Comm. on Solid Physics, 12, 17 (1985).

2. M. Onellion, M.W. Han, F.B. Dunning and G.K. Walters, Phys. Rev. Lett., 52, 380 (1984).

3. M.S. Hammond, F. B. Dunning, G. K. Walters and G.A. Prinz, Phys. Rev B, 45, 3674 (1992).

4. T.W. Riddle, M. Onellion, F.B. Dunning and G.K. Walters, Rev. Sci. Instrum., 52, 797 (1981). 
5. F.-C. Tang, X. Zhang, F.B. Dunning and G.K. Walters, Rev. Sci. Instrum., 59, 504 (1988).

6. D.R. Penn and P. Apell, Phys. Rev. B, 41, 3303 (1990).

7. E. Wimmer, A.J.Freeman and H. Krakauer, Phys. Rev. B, 30, 3113 (1984).

8. C. Rau, J. Magn. Magn. Mater., 30, 141 (1982).

9. R. Wu and A.J. Freeman, Phys. Rev. Lett., 69, 2867 (1992).

10. M.W. Hart, M.S. Hammond, F. B. Dunning and G.K. Walters, Phys. Rev. B, 39, 5488 (1989).

11. D.M. Oró, Q. Lin, P.A. Soletsky, X. Zhang, F.B. Dunning and G.K. Walters, Phys. Rev. B, 46, 9893 (1992).

12. E. Kisker, W. Gudat and K.S. Schröder, Solid State Commun., 44, 591 (1982).

13. J. Unguris, D. T. Pierce, A. Galejs and R.J. Celotta, Phys. Rev. Lett., 49, 72 (1982).

14. J. Kirschner, K. Koike and H.P. Oepen, Vacuum, 41, 518 (1990); Phys. Rev. Lett., 59, 2099 (1987).

15. C. Rau, Appl. Phys. A49, 579 (1989).

16. C. Rau, K. Waters, and N. Chen, Phys. Rev. Lett., 64, 1441 (1990).

17. C. Rau, J. Magn. Magn. Mater., 30, 141 (1982).

18. C. Rau and S. Eichner, Phys. Rev. Lett., 47, 939 (1981).

19. C. Rau, C. Jin and M. Robert, Phys. Lett. A, 138, 334 (1989).

17. C. Rau, P. Mahavadi, and M. Lu, J. Appl. Phys. (1993), in print.

18. N. Yang, Phys. Rev., 85, 808 (1952).

19. C. Rau, N. J. Zheng and M. Lu, J. Magn. Magn. Mat., (1993), in print.

20. J. Balberg and J. S. Helman, Phys. Rev. B, 18, 303 (1978).

21. S. F. Alvarado, M. Campagna, and H. Hopster, Phys. Rev. Lett., 48, 51 (1982); B. H. Dauth, S. F. Alvarado, and M. Campagna, Phys. Rev. Lett., 58, 2118 (1987).

22. C. Rau, G. Xing, C. Liu and M. Robert, Phys. Lett., 135, 227 (1989).

23. H. W. Diehl and E. Eisenriegler, Phys. Rev. B, 30, 300 (1984).

24. J. Quinn, Y.S. Li, H. Li, D. Tian, F. Jona and P.M. Marcus, Phys. Rev. B, 43, 3959 (1991).

25. J. Kirschner, in Surface and Interface Characterization by Electron Optical Methods, ed. by A. Howie and U. Valdre (Plenum Publ. Co., 1988), p. 297.

26. J. Kirschner, K. Koike, and H.P. Oepen, Phys. Rev. Lett., 59 (1987) 2099. 
27. M. Landolt, in "Polarized Electrons in Surface Physics", ed. by R. Feder (World Sci. Publ. Co., 1985), Chap.9; and Refs. cited therein.

28. R.A. Baragiola, E.V. Alonso, and A. Oliva-Florio, Phys. Rev. B61, 121 (1979).

29. C.L. Fu, A.J. Freeman, and T. Oguchi, Phys. Rev. Lett. 54, 2700 (1985).

30. J.W. Krewer and R. Feder, Physica B (1991) 135; and Refs. cited therein.

31. D. Penn and P. Apell, and S.M. Girvin, Phys. Rev. Lett. 55, 518 (1985).

32. J. Glaser and E. Tosatti, Sol. Stat. Comm. 52, 905 (1984). 